Article

\title{
New Mineral with Modular Structure Derived from Hatrurite from the Pyrometamorphic Rocks of the Hatrurim Complex: Ariegilatite, $\mathrm{BaCa}_{12}\left(\mathrm{SiO}_{4}\right)_{4}\left(\mathrm{PO}_{4}\right)_{2} \mathrm{~F}_{2} \mathrm{O}$, from Negev Desert, Israel
}

\author{
Evgeny V. Galuskin ${ }^{1, *}$, Biljana Krüger ${ }^{2}$ (i) , Irina O. Galuskina ${ }^{1}$, Hannes Krüger ${ }^{2}$ (i), \\ Yevgeny Vapnik ${ }^{3}$, Justyna A. Wojdyla ${ }^{4}$ and Mikhail Murashko ${ }^{5}$ \\ 1 Faculty of Earth Sciences, Department of Geochemistry, Mineralogy and Petrography, University of Silesia, \\ Będzińska 60, 41-200 Sosnowiec, Poland; irina.galuskina@us.edu.pl \\ 2 Institute of Mineralogy and Petrography, University of Innsbruck, Innrain 52, 6020 Innsbruck, Austria; \\ biljana.kruger@gmail.com (B.K.); hannes.krueger@gmail.com (H.K.) \\ 3 Department of Geological and Environmental Sciences, Ben-Gurion University of the Negev, \\ P.O. Box 653, Beer-Sheva 84105, Israel; vapnik@bgu.ac.il \\ 4 Swiss Light Source, Paul Scherrer Institute, 5232 Villigen, Switzerland; justyna.wojdyla@psi.ch \\ 5 Saint Petersburg State University, Faculty of Geology, 7-9 Universitetskaya nab., \\ St. Petersburg 199034, Russia; mzmurashko@gmail.com \\ * Correspondence: evgeny.galuskin@us.edu.pl; Tel.: +48-32-3689365
}

Received: 19 February 2018; Accepted: 5 March 2018; Published: 8 March 2018

\begin{abstract}
Ariegilatite, $\mathrm{BaCa}_{12}\left(\mathrm{SiO}_{4}\right)_{4}\left(\mathrm{PO}_{4}\right)_{2} \mathrm{~F}_{2} \mathrm{O}\left(R \overline{3} m, a=7.1551(6) \AA, c=41.303(3) \AA, V=1831.2(3) \AA^{3}\right.$, $Z=3)$, is a new member of the nabimusaite group exhibiting a modular intercalated antiperovskite structure derived from hatrurite. It was found in a few outcrops of pyrometamorphic rocks of the Hatrurim Complex located in the territories of Israel, Palestine and Jordan. The holotype specimen is an altered spurrite marble from the Negev Desert near Arad city, Israel. Ariegilatite is associated with spurrite, calcite, brownmillerite, shulamitite, $\mathrm{CO}_{3}$-bearing fluorapatite, fluormayenite-fluorkyuygenite and a potentially new mineral, $\mathrm{Ba}_{2} \mathrm{Ca}_{18}\left(\mathrm{SiO}_{4}\right)_{6}\left(\mathrm{PO}_{4}\right)_{3}\left(\mathrm{CO}_{3}\right) \mathrm{F}_{3} \mathrm{O}$. Ariegilatite is overgrown and partially replaced by stracherite, $\mathrm{BaCa}_{6}\left(\mathrm{SiO}_{4}\right)_{2}\left[\left(\mathrm{PO}_{4}\right)\left(\mathrm{CO}_{3}\right)\right] \mathrm{F}$. The mineral forms flat disc-shaped crystals up to $0.5 \mathrm{~mm}$ in size. It is colorless, transparent, with white steaks and vitreous luster. Optically, ariegilatite is uniaxial, negative: $\omega=1.650(2), \varepsilon=1.647(2)(\lambda=589 \mathrm{~nm})$. The mean composition of the holotype ariegilatite, $\left(\mathrm{Ba}_{0.98} \mathrm{~K}_{0.01} \mathrm{Na}_{0.01}\right)_{\Sigma 1}\left(\mathrm{Ca}_{11.77} \mathrm{Na}_{0.08} \mathrm{Fe}^{2+}{ }_{0.06} \mathrm{Mn}^{2+}{ }_{0.05} \mathrm{Mg}_{0.04}\right)_{\Sigma 12}\left(\mathrm{Si}_{3.95} \mathrm{Al}_{0.03} \mathrm{Ti}_{0.02}\right)_{\Sigma 4}\left(\mathrm{P}_{1.70} \mathrm{C}_{0.16} \mathrm{Si}_{0.10} \mathrm{~S}^{6+}{ }_{0.03}\right.$ $\left.\mathrm{V}_{0.01}\right)_{\Sigma 2} \mathrm{~F}_{2.04} \mathrm{O}_{0.96}$, is close to the end-member formula. The structure of ariegilatite is described as a stacking of the two modules $\left\{\mathrm{F}_{2} \mathrm{OCa}_{12}\left(\mathrm{SiO}_{4}\right)_{4}\right\}^{4+}$ and $\left\{\mathrm{Ba}\left(\mathrm{PO}_{4}\right)_{2}\right\}^{4-}$ along (001). Ariegilatite, as well as associated stracherite, are high-temperature alteration products of minerals of an early clinker-like association. These alterations took place under the influence of pyrometamorphism by-products, such as gases and fluids generated by closely-spaced combustion foci.
\end{abstract}

Keywords: ariegilatite; nabimusaite group; new mineral; crystal structure; intercalated hexagonal antiperovskite; $\mathrm{CO}_{3}$-group; Raman; pyrometamorphic rocks; Hatrurim Complex

\section{Introduction}

Ariegilatite, $\mathrm{BaCa}_{12}\left(\mathrm{SiO}_{4}\right)_{4}\left(\mathrm{PO}_{4}\right)_{2} \mathrm{~F}_{2} \mathrm{O}\left(R \overline{3} m, a=7.1551(6) \AA, c=41.303(3) \AA, V=1831.2(3) \AA^{3}\right.$, $\mathrm{Z}=3$ ), is the first P-bearing mineral in the nabimusaite group, which combines nabimusaite, $\mathrm{KCa}_{12}\left(\mathrm{SiO}_{4}\right)_{4}\left(\mathrm{SO}_{4}\right)_{2} \mathrm{O}_{2} \mathrm{~F}\left(R \overline{3} m, a=7.1905(4), c=41.251(3) \AA, V=1847.1(2) \AA^{3}, \mathrm{Z}=3\right)$ and dargaite, $\mathrm{BaCa}_{12}\left(\mathrm{SiO}_{4}\right)_{4}\left(\mathrm{SO}_{4}\right)_{2} \mathrm{O}_{3}\left(R \overline{3} m, a=7.1874(4) ; c=41.292(3) \AA ; V=1847.32(19) \AA^{3}, \mathrm{Z}=3\right)[1,2]$. Ariegilatite 
was found in altered spurrite marble belonging to the pyrometamorphic Hatrurim Complex in the Negev Desert near Arad, Israel. Rocks of the Hatrurim Complex mainly consist of spurrite marbles, larnite pseudoconglomerates and gehlenite hornfelses. Furthermore, pyrometamorphic rocks and by-products of their low-temperature alterations commonly occur along the Dead Sea Rift in the territories of Israel, Palestine and Jordan (the "Mottled zone") [3-5].

Minerals of the nabimusaite group are silicates with additional anions and are isostructural with arctite, a phosphate with an ideal crystal chemical formula of $\mathrm{Ba}\left(\mathrm{Ca}_{7} \mathrm{Na}_{5}\right)\left(\mathrm{PO}_{4}\right)_{6} \mathrm{~F}_{3}$ [6]. Minerals of the nabimusaite group and arctite can be described as hexagonal intercalated antiperovskites with the general crystal chemical formula $A B_{12}\left(T \mathrm{O}_{4}\right)_{4}\left(T \mathrm{O}_{4}\right)_{2} W_{3}, A=\mathrm{Ba}, \mathrm{K}, \ldots ; B=\mathrm{Ca}, \mathrm{Na}, \ldots$; $T=\mathrm{Si}, \mathrm{P} \mathrm{S}^{6+}, \mathrm{V}^{5+}, \mathrm{Al}, \ldots ; W=\mathrm{O}^{2-}, \mathrm{F}^{-}[2,7]$. Nabimusaite group minerals show hatrurite-like triple antiperovskite layers $\left\{\left[W_{3} \mathrm{Ca}_{12}\right]\left(\mathrm{SiO}_{4}\right)_{4}\right\}^{\mathrm{e}+}$, which alternate with $A\left(\mathrm{TO}_{4}\right)_{2}{ }^{\mathrm{e}-}$ single layers $[1,2]$. The same type of layers is found in arctite. However, the composition of the triple antiperovskite module is $\left\{\left[\mathrm{F}_{3}\left(\mathrm{Ca}_{7} \mathrm{Na}_{5}\right)\right]\left(\mathrm{PO}_{4}\right)_{4}\right\}^{4+}$, and the second module is $\mathrm{Ba}\left(\mathrm{PO}_{4}\right)_{2}{ }^{4-}[6,8]$.

Minerals of the zadovite group can also be described as intercalated antiperovskites. Their general formula is $A B_{6}\left(\mathrm{TO}_{4}\right)_{2}\left[\left(\mathrm{TO}_{4}\right)_{2-\mathrm{x}}\left(\mathrm{CO}_{3}\right)_{\mathrm{x}}\right] W(A, B, T, W=$ as above; $\mathrm{x} \approx 0$ or $\mathrm{x} \approx 1)$ : zadovite, $\mathrm{BaCa}_{6}\left[\left(\mathrm{SiO}_{4}\right)\left(\mathrm{PO}_{4}\right)\right]\left(\mathrm{PO}_{4}\right)_{2} \mathrm{~F}$; aradite, $\mathrm{BaCa}_{6}\left[\left(\mathrm{SiO}_{4}\right)\left(\mathrm{VO}_{4}\right)\right]\left(\mathrm{VO}_{4}\right)_{2} \mathrm{~F}$; gazeevite, $\mathrm{BaCa}_{6}\left(\mathrm{SiO}_{4}\right)_{2}\left(\mathrm{SO}_{4}\right)_{2} \mathrm{O}$ and stracherite $\mathrm{BaCa}_{6}\left(\mathrm{SiO}_{4}\right)_{2}\left[\left(\mathrm{PO}_{4}\right)\left(\mathrm{CO}_{3}\right)\right] \mathrm{F}$. In these minerals, single antiperovskite layers $\left\{\left[\mathrm{WB}_{6}\right]\left(\mathrm{TO}_{4}\right)_{2}\right\}^{\mathrm{e}+}$ intercalate with single $\mathrm{Ba}\left(\mathrm{TO}_{4}\right)_{2}{ }^{\mathrm{e}-}$ layers [7,9]. In stracherite, about half of the $\left(\mathrm{PO}_{4}\right)^{3-}$ tetrahedra are replaced by $\left(\mathrm{CO}_{3}\right)^{2-}$ groups [10].

Ariegilatite is named in honor of Dr. Arie Gilat (b. 1939). Arie Gilat is retired from the Geological Survey of Israel, where he was involved in geological mapping, tectonics and geochemical studies for more than 30 years. He is the author and co-author of numerous geological papers. At present, his main interests are related to the study of earthquake physics and processes at the core and mantle interface. His continuous support, consulting and several new unconventional ideas on the genesis of the Hatrurim Complex are greatly appreciated by the authors.

Ariegilatite was approved as a new mineral species by Commission on New Minerals, Nomenclature and Classification, International Mineralogical Association (CNMNC IMA) in March 2017 (IMA2016-100). The material was deposited in the mineralogical collection of the Fersman Mineralogical Museum, Leninskiy pr., 18/k2, 115162 Moscow, Russia, Catalogue Number 4956/1.

\section{Methods of Investigation}

The crystal morphology and chemical composition of ariegilatite and associated minerals were examined using optical microscopes, as well as the analytical electron scanning microscopes Philips XL30 and Phenom XL, PhenomWorld, ThermoFisher Scientific, Eindhoven, The Netherlands (Faculty of Earth Sciences, University of Silesia, Sosnowiec, Poland). Chemical analyses of ariegilatite were performed with a CAMECA SX100 microprobe analyzer (Institute of Geochemistry, Mineralogy and Petrology, University of Warsaw, Warszawa, Poland) at $15 \mathrm{kV}$ and $10 \mathrm{nA}$ using the following lines and standards: $\mathrm{Ba} L \alpha, \mathrm{SK} \alpha$, baryte; $\mathrm{PK} \alpha$, apatite; $\mathrm{CaK} \alpha$, wollastonite; $\mathrm{Mg} K \alpha$, SiK $\alpha$, diopside; FeK $\alpha$, hematite, $\mathrm{Al} K \alpha, \mathrm{K} K \alpha$, orthoclase; $\mathrm{Ti} K \alpha$, rutile; $\mathrm{NaK} \alpha$, albite, $\mathrm{Sr} L \alpha, \mathrm{SrTiO}_{3}, \mathrm{FK} \alpha$, fluorphlogopite.

Raman spectra of ariegilatite were recorded on a WITec alpha 300R Confocal Raman Microscope, WITec, Ulm, Germany (Department of Earth Science, University of Silesia, Sosnowiec, Poland) equipped with an air-cooled solid-state laser $(532 \mathrm{~nm})$ and a CCD camera operating at $-61{ }^{\circ} \mathrm{C}$. The laser radiation was coupled to a microscope through a single-mode optical fiber with a diameter of $3.5 \mu \mathrm{m}$. An air Zeiss LD EC Epiplan-Neofluan DIC-100/0.75NA objective was used. Raman scattered light was focused on a broad band single mode fiber with an effective pinhole size of about $30 \mu \mathrm{m}$, and a monochromator with a $600-\mathrm{mm}^{-1}$ grating was used. The power of the laser at the sample position was ca. $40 \mathrm{~mW}$. Integration times of $5 \mathrm{~s}$ with an accumulation of 15-20 scans and a resolution $3 \mathrm{~cm}^{-1}$ were chosen. The monochromator was calibrated using the Raman scattering line of a silicon plate $\left(520.7 \mathrm{~cm}^{-1}\right)$. Fitting of spectra was performed with the help of the "GRAMS" program using the mixed Lorentz-Gauss function. 
Single-crystal X-ray diffraction data were collected from a crystal of ariegilatite $(\sim 38 \times 32 \times 25 \mu \mathrm{m})$ using synchrotron radiation at the super-bending magnet beamline X06DA at the Swiss Light Source, Paul Scherrer Institute, Villigen, Switzerland. The multi-axis goniometer PRIGo [11] and a PILATUS 2M-F detector, which was placed at a distance of $120 \mathrm{~mm}$ from the crystal with a vertical offset of $67 \mathrm{~mm}$, was used. The wavelength was set to $\lambda=0.70848 \AA$. The data were collected in a single $180^{\circ}$ omega scan with steps of $0.1^{\circ}$ and 0.1 -s exposures, controlled by the DA + software [12] Data evaluation and processing was performed using the CrysAlisPro software package [13]. As a starting model, the structure of the isostructural analogue nabimusaite was used, adapted to the expected composition. With subsequent analyses of difference-Fourier maps, the structure was refined to $R_{1}=1.95 \%$. The refinements include anisotropic displacement-parameters and have been carried out with neutral atom scattering-factors, using the program SHELX97 [14].

\section{Results}

\subsection{Occurrence and Description of Holotype Specimen}

Ariegilatite was found in a few outcrops of pyrometamorphic rocks of the Hatrurim Complex located in the territories of Israel, Palestine and Jordan. Investigations of a new mineral were performed in samples of spurrite rocks collected in the Negev Desert (Hatrurim Basin, N31 $13^{\prime}$ E35 $\left.{ }^{\circ} 16^{\prime}\right)$ near Arad, Israel. Ariegilatite forms strongly flattened crystals of disc-shaped form. In thin-sections, its pseudo-aciculate morphology is usually observed (Figure 1A). The size of some highly-fractured crystals of ariegilatite reaches $0.5 \mathrm{~mm}$ with a thickness of $0.1 \mathrm{~mm}$ (Figure 1A). Ariegilatite is associated with spurrite, calcite, brownmillerite, shulamitite, $\mathrm{CO}_{3}$-bearing fluorapatite, fluormayenite-fluorkyuygenite, periclase, brucite, barytocalcite, baryte, garnets of elbrusite-kerimasite series, unidentified $\mathrm{Ca}-\mathrm{Fe}$ - and $\mathrm{Rb}$-bearing $\mathrm{K}-\mathrm{Fe}$ sulfides and a potentially new mineral, $\mathrm{Ba}_{2} \mathrm{Ca}_{18}\left(\mathrm{SiO}_{4}\right)_{6}\left(\mathrm{PO}_{4}\right)_{3}\left(\mathrm{CO}_{3}\right) \mathrm{F}_{3} \mathrm{O}$ [15]. Ariegilatite is often overgrown and replaced by stracherite (Figure $1 \mathrm{~B}$ ). Ariegilatite and stracherite are usually limited to re-crystallization zones of dark-grey fine-grained spurrite rocks, which differ from the surrounding rocks by discoloration, development of thin calcite veins and also by local appearance of large spurrite metacrysts (up to $1 \mathrm{~cm}$ in size), as well as the presence of sulfide mineralization.
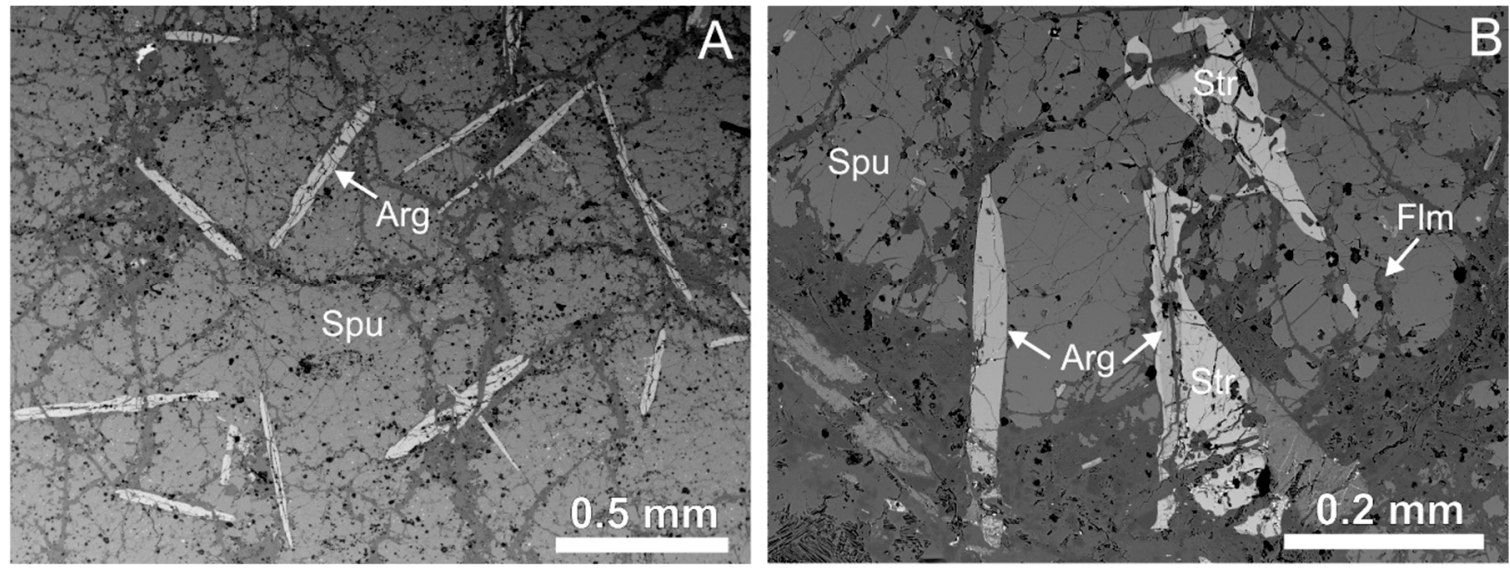

Figure 1. Disc-shaped, flattened ariegilatite crystals in spurrite rock (A), holotype specimen L15; overgrowing and replacing of ariegilatite by stracherite (B). Arg = ariegilatite, Flm = fluormayenite, Spu = spurrite, $\operatorname{Str}=$ stracherite.

Ariegilatite is a colorless, transparent mineral with white streaks and vitreous luster. It does not show any fluorescence. The mineral is uniaxial, negative: $\omega=1.650(2), \varepsilon=1.647(2)(\lambda=589 \mathrm{~nm})$ and non-pleochroic. The hardness was measured using micro-indentation and determined to $\mathrm{VHN}_{50}=356(16) \mathrm{kg} \cdot \mathrm{mm}^{-2}$, as an average of 16 measured spots ranging from $331-378 \mathrm{~kg} \cdot \mathrm{mm}^{-2}$, which 
corresponds to 4-4.5 on the Mohs scale. In contrast to minerals of the nabimusaite-dargaite series [1,2], ariegilatite does not show pronounced cleavage on (001), and the fracture is irregular. The small size of separated crystal fragments does not allow measuring the density. Therefore, the density was calculated on the basis of structural data and the mean composition of ariegilatite: $3.329 \mathrm{~g} \cdot \mathrm{cm}^{-3}$. Compatibility index $1-\left(\mathrm{K}_{\mathrm{p}} / \mathrm{K}_{\mathrm{c}}\right)=-0.017$ (superior) was calculated for the empirical formula of ariegilatite of the holotype specimen (Table 1 ).

The Raman spectrum of ariegilatite exhibits the following strong bands (Figure 2; $\mathrm{cm}^{-1}$ ): 129, 179, 229 and 309 (lattice mode, Ba-O, Ca-O vibrations); $403\left[v_{2}\left(\mathrm{SiO}_{4}\right)^{4-}\right] ; 427\left[v_{2}\left(\mathrm{PO}_{4}\right)^{2-}\right] ; 520\left[v_{4}\left(\mathrm{SiO}_{4}\right)^{4-}\right]$; 569 and $591\left[v_{4}\left(\mathrm{PO}_{4}\right)^{3-}\right] ; 834$ and $\left.874\left[v_{1}\left(\mathrm{SiO}_{4}\right)^{4-}\right] ; 947\left[v_{1}\left(\mathrm{PO}_{4}\right)^{3-}\right] ; 993\left[v_{1} \mathrm{SO}_{4}\right)^{2-}\right] ; 1030\left[v_{3}\left(\mathrm{PO}_{4}\right)^{2-}\right]$; $1066\left[v_{1}\left(\mathrm{CO}_{3}\right)^{2-}\right]$. The main strong Raman bands of ariegilatite are related to vibrations in $\left(\mathrm{SiO}_{4}\right)^{4-}$ and $\left(\mathrm{PO}_{4}\right)^{3-}$ (Figure 2$)$. The band at about $1066 \mathrm{~cm}^{-1}$ can be attributed to $v_{1}\left(\mathrm{CO}_{3}\right)^{2-}$. Its position is very close to the $v_{1}\left(\mathrm{CO}_{3}\right)^{2-}$ mode in $\mathrm{CO}_{3}$-bearing hydroxylapatite and hydroxylellestadite [16,17]. Raman spectroscopy data indicate that $\mathrm{H}_{2} \mathrm{O}$ is absent in ariegilatite. Direct determination of the $\mathrm{CO}_{2}$ content was not feasible due to abundant microscopic inclusions of other minerals.

The mean composition of the holotype ariegilatite, $\left(\mathrm{Ba}_{0.98} \mathrm{~K}_{0.01} \mathrm{Na}_{0.01}\right)_{\Sigma 1}\left(\mathrm{Ca}_{11.77} \mathrm{Na}_{0.08} \mathrm{Fe}^{2+}{ }_{0.06} \mathrm{Mn}^{2+}{ }_{0.05}\right.$ $\left.\mathrm{Mg}_{0.04}\right)_{\Sigma 12}\left(\mathrm{Si}_{3.95} \mathrm{Al}_{0.03} \mathrm{Ti}_{0.02}\right)_{\Sigma 4}\left(\mathrm{P}_{1.70} \mathrm{C}_{0.16} \mathrm{Si}_{0.10} \mathrm{~S}^{6+}{ }_{0.03} \mathrm{~V}_{0.01}\right)_{\Sigma 2} \mathrm{~F}_{2.04} \mathrm{O}_{0.96}$, is close to the end-member formula $\mathrm{BaCa}_{12}\left(\mathrm{SiO}_{4}\right)_{4}\left(\mathrm{PO}_{4}\right)_{2} \mathrm{~F}_{2} \mathrm{O}$. The amount of $\mathrm{CO}_{3}$ in ariegilatite is calculated assuming charge balance and 13 non-tetrahedral cations (Table 1, L15).

Experimental details and refinement data of ariegilatite are summarized in Tables 2-5. The structural formula of ariegilatite $\mathrm{Ba} 1 \mathrm{Ca}{ }_{6} \mathrm{Ca}_{6}\left[\left(\mathrm{Si}_{1} \mathrm{O}_{4}\right)_{2}\left(\mathrm{Si} 2 \mathrm{O}_{4}\right)_{2}\right]\left(\mathrm{P} 1 \mathrm{O}_{4}\right)_{\sim 1.8} \mathrm{~F}_{2} \mathrm{O}_{2}$ (Table 4) is simplified to $\mathrm{BaCa}_{12}\left(\mathrm{SiO}_{4}\right)_{4}\left(\mathrm{PO}_{4}\right)_{2} \mathrm{~F}_{2} \mathrm{O}$ according to the general formula of the nabimusaite group minerals [2]. The ariegilatite structure is most easily described as a 1:1 stacking of the two modules $\left\{\left[\mathrm{F}_{2} \mathrm{OCa}_{12}\right]\left(\mathrm{SiO}_{4}\right)_{4}\right\}^{4+}$ and $\left\{\mathrm{Ba}\left(\mathrm{PO}_{4}\right)_{2}\right\}^{4-}$ along (001) (Figure 3). The sites $\mathrm{F} 1$ and $\mathrm{O} 7$ are coordinated by six $\mathrm{Ca}$ atoms in an octahedral arrangement (Figure $3 \mathrm{~B})$. The module $\left\{\mathrm{Ba}\left(\mathrm{PO}_{4}\right)_{2}\right\}^{4-}$ is characterized by $\left(\mathrm{P} \mathrm{O}_{4}\right)$ tetrahedra connected to six-coordinated Ba1 (Figure 3C).

Table 1. Chemical composition of ariegilatite, wt \%.

\begin{tabular}{|c|c|c|c|c|c|c|c|c|c|}
\hline \multirow[b]{2}{*}{ Mean } & \multicolumn{3}{|c|}{ L15 } & \multicolumn{3}{|c|}{ YV595 } & \multicolumn{3}{|c|}{ SS20b } \\
\hline & 22 & s.d. & Range & 23 & s.d. & Range & 14 & s.d. & Range \\
\hline $\mathrm{SO}_{3}$ & 0.17 & 0.07 & $0.05-0.31$ & 0.60 & 0.22 & $0.32-1.02$ & n.d. & & \\
\hline $\mathrm{V}_{2} \mathrm{O}_{5}$ & 0.10 & 0.06 & $0-0.17$ & 0.32 & 0.08 & $0.13-0.51$ & 0.41 & 0.07 & $0.26-0.52$ \\
\hline $\mathrm{P}_{2} \mathrm{O}_{5}$ & 9.83 & 0.45 & $8.96-10.55$ & 10.39 & 0.55 & $9.63-12.53$ & 10.61 & 0.4 & $10.05-11.40$ \\
\hline $\mathrm{TiO}_{2}$ & 0.12 & 0.06 & $0.05-0.25$ & 0.31 & 0.04 & $0.24-0.40$ & 0.26 & 0.16 & $0.10-0.56$ \\
\hline $\mathrm{SiO}_{2}$ & 19.87 & 0.26 & $19.52-20.42$ & 19.21 & 0.36 & $17.70-19.62$ & 18.64 & 0.27 & $18.21-19.15$ \\
\hline $\mathrm{Fe}_{2} \mathrm{O}_{3}$ & & & & 0.34 & 0.09 & & 0.17 & 0.05 & $0.11-0.24$ \\
\hline $\mathrm{Al}_{2} \mathrm{O}_{3}$ & 0.12 & 0.03 & $0.07-0.18$ & 0.17 & 0.11 & $0.10-0.68$ & 0.24 & 0.06 & $0.17-0.38$ \\
\hline $\mathrm{BaO}$ & 12.26 & 0.06 & $12.14-12.41$ & 12.01 & 0.35 & $10.81-12.49$ & 12.29 & 0.34 & $11.74-12.94$ \\
\hline $\mathrm{FeO}$ & 0.32 & 0.06 & $0.24-0.46$ & 0.49 & & & & & \\
\hline $\mathrm{MnO}$ & 0.29 & 0.08 & $0.09-0.39$ & 0.26 & 0.07 & $0.14-0.41$ & n.d. & & \\
\hline $\mathrm{CaO}$ & 53.84 & 0.24 & $53.19-54.40$ & 53.77 & 0.30 & $53.13-54.27$ & 54.24 & 0.31 & $54.30-55.62$ \\
\hline $\mathrm{MgO}$ & 0.14 & 0.03 & $0.11-0.22$ & 0.38 & 0.05 & $0.27-0.53$ & 0.03 & 0.02 & $0-0.07$ \\
\hline $\mathrm{K}_{2} \mathrm{O}$ & 0.04 & 0.03 & $0-0.10$ & n.d. & & & n.d. & & \\
\hline $\mathrm{Na}_{2} \mathrm{O}$ & 0.22 & 0.05 & $0.16-0.36$ & 0.05 & 0.02 & $0.02-0.09$ & 0.31 & 0.05 & $0.20-0.38$ \\
\hline $\mathrm{F}$ & 3.17 & 0.10 & $2.96-3.34$ & 3.24 & 0.45 & $2.27-3.27$ & 3.05 & 0.11 & $2.84-3.17$ \\
\hline $\mathrm{CO}_{2} *$ & 0.57 & & & 0.00 & & & 0.62 & & \\
\hline$-\mathrm{O}=\mathrm{F}$ & 1.33 & & & 1.36 & & & 1.28 & & \\
\hline Total & 99.72 & & & 100.18 & & & 99.58 & & \\
\hline $\mathrm{Ba}$ & 0.98 & & & 0.96 & & & 0.99 & & \\
\hline K & 0.01 & & & & & & & & \\
\hline $\mathrm{Na}$ & 0.01 & & & 0.02 & & & 0.01 & & \\
\hline $\mathrm{Ca}$ & & & & 0.02 & & & & & \\
\hline A & 1 & & & 1 & & & 1 & & \\
\hline $\mathrm{Ca}$ & 11.77 & & & 11.75 & & & 11.88 & & \\
\hline $\mathrm{Mn}^{2+}$ & 0.05 & & & 0.05 & & & & & \\
\hline $\mathrm{Fe}^{2+}$ & 0.06 & & & 0.08 & & & & & \\
\hline $\mathrm{Mg}$ & 0.04 & & & 0.12 & & & 0.01 & & \\
\hline $\mathrm{Na}$ & 0.08 & & & & & & 0.11 & & \\
\hline
\end{tabular}


Table 1. Cont.

\begin{tabular}{|c|c|c|c|c|c|c|c|c|c|c|}
\hline \multirow[b]{2}{*}{ Mean } & \multicolumn{3}{|c|}{ L15 } & \multicolumn{3}{|c|}{ YV595 } & \multicolumn{4}{|c|}{ SS20b } \\
\hline & 22 & s.d. & Range & 23 & s.d. & Range & 14 & s.d. & Rang & \\
\hline B & 12 & & & 12 & & & 12 & & & \\
\hline $\mathrm{Si}$ & 3.95 & & & 3.86 & & & 3.81 & & & \\
\hline $\mathrm{Ti}^{4+}$ & 0.02 & & & 0.05 & & & 0.04 & & & \\
\hline $\mathrm{Fe}^{3+}$ & & & & 0.05 & & & 0.03 & & & \\
\hline $\mathrm{Al}$ & 0.03 & & & 0.04 & & & 0.06 & & & \\
\hline $\mathrm{P}$ & & & & & & & 0.07 & & & \\
\hline T1 & 4 & & & 4 & & & 4 & & & \\
\hline $\mathrm{P}$ & 1.70 & & & 1.80 & & & 1.77 & & & \\
\hline $\mathrm{Si}$ & 0.10 & & & 0.07 & & & & & & \\
\hline $\mathrm{V}^{5+}$ & 0.01 & & & 0.04 & & & 0.06 & & & \\
\hline $\mathrm{S}^{6+}$ & 0.03 & & & 0.09 & & & & & & \\
\hline $\mathrm{C}$ & 0.16 & & & & & & 0.17 & & & \\
\hline T2 & 2 & & & 2 & & & 2 & & & \\
\hline $\mathrm{O}$ & 0.96 & & & 0.91 & & & 1.03 & & & \\
\hline $\mathrm{F}$ & 2.04 & & & 2.09 & & & 1.97 & & & \\
\hline \multirow[t]{2}{*}{ W } & 2 & & & 2 & & & 2 & & & \\
\hline & \multicolumn{3}{|c|}{ SS27A } & \multicolumn{3}{|c|}{ MA5b } & \multicolumn{3}{|c|}{ IS129 } & teor \\
\hline Mean & 29 & s.d. & Range & 11 & s.d. & Range & 12 & s.d. & Range & \\
\hline $\mathrm{SO}_{3}$ & 0.05 & 0.08 & $0-0.34$ & 1.35 & 0.22 & $1.10-1.92$ & n.d. & & & \\
\hline $\mathrm{V}_{2} \mathrm{O}_{5}$ & 0.42 & 0.07 & $0.24-0.62$ & 0.18 & 0.11 & $0.04-0.41$ & n.d. & & & \\
\hline $\mathrm{P}_{2} \mathrm{O}_{5}$ & 10.52 & 0.52 & $9.48-11.65$ & 12.91 & 0.43 & $11.89-13.60$ & 9.02 & 0.49 & $8.31-10.16$ & 11.53 \\
\hline $\mathrm{TiO}_{2}$ & 0.27 & 0.16 & $0.05-0.59$ & 0.18 & 0.06 & $0.09-0.28$ & n.d. & & & \\
\hline $\mathrm{SiO}_{2}$ & 18.85 & 0.42 & $18.25-20.30$ & 16.46 & 0.45 & $15.81-17.16$ & 19.83 & 0.27 & $19.44-20.23$ & 19.53 \\
\hline $\mathrm{Fe}_{2} \mathrm{O}_{3}$ & n.d. & & & & & & n.d. & & & \\
\hline $\mathrm{Al}_{2} \mathrm{O}_{3}$ & 0.25 & 0.08 & $0.14-0.48$ & 0.53 & 0.10 & $0.40-0.75$ & 0.15 & 0.1 & $0.09-0.47$ & \\
\hline $\mathrm{BaO}$ & 12.26 & 0.41 & $11.39-13.15$ & 12.17 & 0.49 & $11.42-13.31$ & 12.36 & 0.17 & $12.12-12.66$ & 12.46 \\
\hline $\mathrm{FeO}$ & & & & 0.17 & 0.11 & $0.03-0.38$ & & & & \\
\hline $\mathrm{MnO}$ & n.d. & & & n.d. & & & n.d. & & & \\
\hline $\mathrm{CaO}$ & 54.26 & 0.36 & $54.00-55.58$ & 54.49 & 0.13 & $54.25-54.68$ & 54.78 & 0.15 & $54.56-55.07$ & 54.68 \\
\hline $\mathrm{MgO}$ & 0.08 & 0.16 & $0-0.93$ & 0.09 & 0.02 & $0.06-0.12$ & n.d. & & & \\
\hline $\mathrm{K}_{2} \mathrm{O}$ & n.d. & & & 0.06 & 0.02 & $0.02-0.10$ & 0.04 & 0.02 & $0.02-0.08$ & \\
\hline $\mathrm{Na}_{2} \mathrm{O}$ & 0.24 & 0.08 & $0.06-0.37$ & 0.23 & 0.04 & $0.15-0.32$ & 0.66 & 0.11 & $0.49-0.82$ & \\
\hline F & 3.02 & 0.15 & $2.68-3.37$ & 2.30 & 0.07 & $2.15-2.48$ & 2.92 & 0.14 & $2.56-3.22$ & 3.08 \\
\hline $\mathrm{CO}_{2} *$ & 0.55 & & & 0.18 & & & 1.68 & & & 0.00 \\
\hline$-\mathrm{O}=\mathrm{F}$ & 1.27 & & & 0.97 & & & 1.23 & & & 1.29 \\
\hline Total & 99.50 & & & 100.32 & & & 100.21 & & & 100.00 \\
\hline $\mathrm{Ba}$ & 0.98 & & & 0.97 & & & 0.97 & & & 1.000 \\
\hline K & & & & 0.02 & & & 0.01 & & & \\
\hline $\mathrm{Na}$ & 0.02 & & & 0.01 & & & 0.02 & & & \\
\hline \multicolumn{11}{|l|}{$\mathrm{Ca}$} \\
\hline A & 1 & & & 1 & & & 1 & & & \\
\hline \multirow{2}{*}{\multicolumn{11}{|c|}{$\mathrm{Mn}^{2+}$}} \\
\hline & & & & & & & & & & \\
\hline $\mathrm{Fe}^{2+}$ & & & & 0.03 & & & & & & \\
\hline $\mathrm{Mg}$ & 0.02 & & & 0.03 & & & & & & \\
\hline $\mathrm{Na}$ & 0.08 & & & 0.07 & & & 0.24 & & & \\
\hline B & 12 & & & 12 & & & 12 & & & \\
\hline $\mathrm{Si}$ & 3.86 & & & 3.35 & & & 3.97 & & & 4.000 \\
\hline $\mathrm{Ti}^{4+}$ & 0.04 & & & 0.02 & & & & & & \\
\hline \multicolumn{11}{|l|}{$\mathrm{Fe}^{3+}$} \\
\hline $\mathrm{Al}$ & 0.06 & & & 0.13 & & & 0.03 & & & \\
\hline $\mathrm{P}$ & 0.04 & & & 0.50 & & & & & & \\
\hline T1 & 4 & & & 4 & & & 4 & & & \\
\hline $\mathrm{P}$ & 1.78 & & & 1.72 & & & 1.53 & & & 2.000 \\
\hline $\mathrm{Si}$ & & & & & & & 0.01 & & & \\
\hline $\mathrm{V}^{5+}$ & 0.06 & & & 0.02 & & & & & & \\
\hline$S^{6+}$ & 0.01 & & & 0.21 & & & & & & \\
\hline $\mathrm{C}$ & 0.15 & & & 0.05 & & & 0.46 & & & \\
\hline T2 & 2 & & & 2 & & & 2 & & & \\
\hline $\mathrm{O}$ & 1.04 & & & 1.52 & & & 1.15 & & & 1.000 \\
\hline $\mathrm{F}$ & 1.96 & & & 1.48 & & & 1.85 & & & 2.000 \\
\hline W & 2 & & & 2 & & & 2 & & & 2 \\
\hline
\end{tabular}




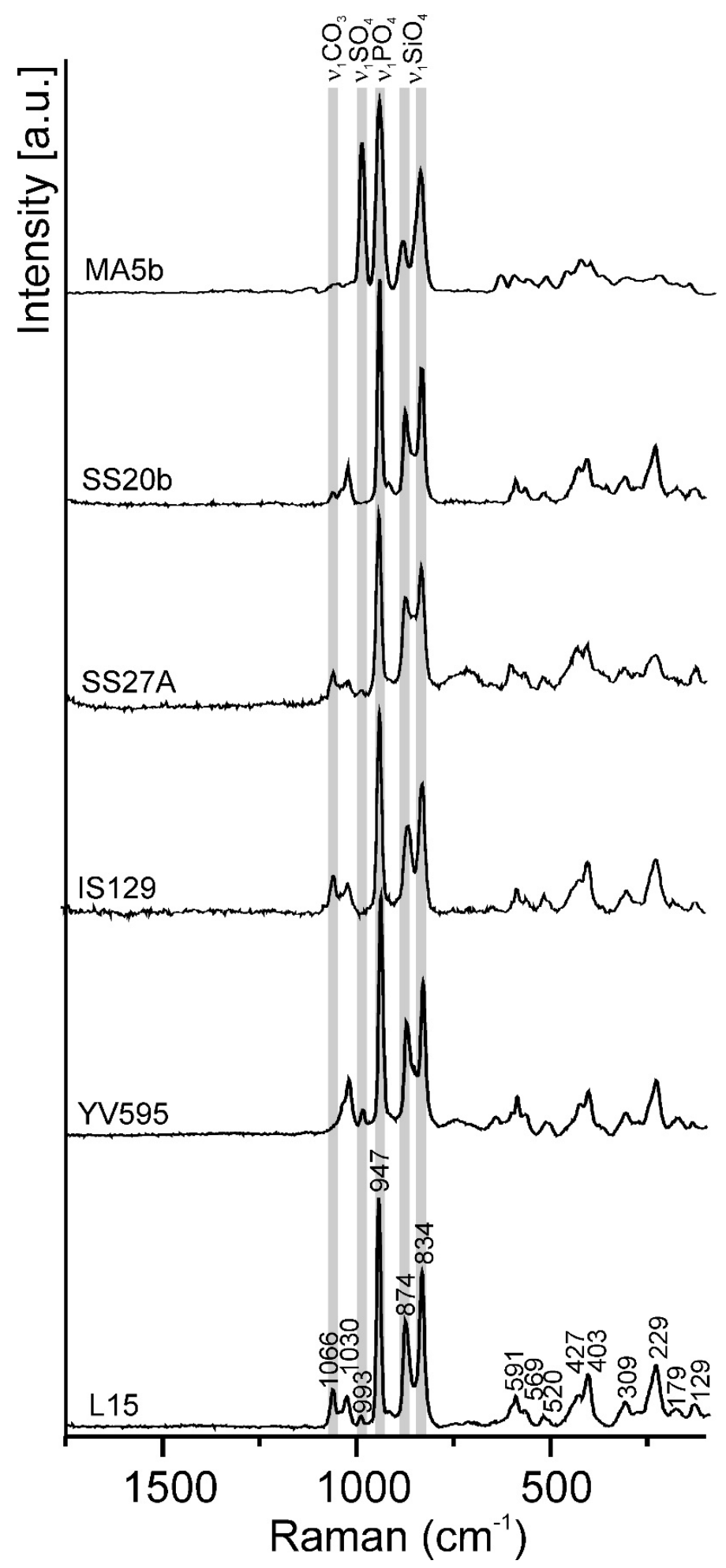

Figure 2. Raman spectra of ariegilatite: holotype specimen, Negev Desert, Israel (Sample Number L15); gehlenite-larnite rock, Daba-Siwaqa, Jordan (YV595); apatite-spurrite rock, Gurim Anticline, Negev Desert (IS129); spurrite rock, Daba-Siwaqa, Jordan (SS27A and SS20b); exsolution structures after flamite (MA5b). Grey vertical bars show the positions of characteristic bands of stretching vibrations $\left(v_{1}\right)$ in tetrahedral anion groups and $\left(\mathrm{CO}_{3}\right)^{2-}$. 
Table 2. Parameters for X-ray data collection.

\begin{tabular}{|c|c|}
\hline \multicolumn{2}{|c|}{ Crystal Data } \\
\hline Crystal system & trigonal \\
\hline Unit cell dimensions ( $\mathrm{A})$ & $\begin{array}{c}a=7.1551(6), c=41.303(3) \AA, b=7.1551(6) \\
c=41.303(3) \\
\alpha, \beta=90^{\circ} \gamma=120^{\circ}\end{array}$ \\
\hline Space group & $R \overline{3} m($ no.166) \\
\hline Volume $\left(\AA^{3}\right)$ & $1831.2(3)$ \\
\hline Z & 3 \\
\hline Density (calculated) & $3.329 \mathrm{~g} \cdot \mathrm{cm}^{-3}$ \\
\hline Chemical formula & $\sim \mathrm{BaCa}_{12}\left(\mathrm{SiO}_{4}\right)_{4}\left(\mathrm{PO}_{4}\right)_{1.8} \mathrm{~F}_{2} \mathrm{O}$ \\
\hline Crystal size $(\mathrm{mm})$ & $38 \times 32 \times 25 \mu \mathrm{m}$ \\
\hline \multicolumn{2}{|c|}{ Data Collection } \\
\hline Diffractometer & $\begin{array}{c}\text { beamline X06DA, SLS } \\
\text { multi-axis goniometer PRIGo } \\
\text { PILATUS 2M-F detector } \\
\lambda=0.70848 \AA\end{array}$ \\
\hline Max. $\theta^{\circ}$-range for data collection & 32.139 \\
\hline Index ranges & $-10 \leq h \leq 6$ \\
\hline & $-8 \leq k \leq 10$ \\
\hline No. of measured reflections & $\begin{array}{c}-39 \leq 1 \leq 44 \\
3445\end{array}$ \\
\hline No. of unique reflections & 825 \\
\hline No. of observed reflections $(\mathrm{I}>2 \sigma(\mathrm{I}))$ & 822 \\
\hline \multicolumn{2}{|c|}{ Refinement of the Structure } \\
\hline No. of parameters used in refinement & 58 \\
\hline Rint & 0.0199 \\
\hline$R \sigma$ & 0.0105 \\
\hline$R 1, I>2 \sigma(\mathrm{I})$ & 0.0195 \\
\hline$R 1$ all Data & 0.0195 \\
\hline $\mathrm{w} R 2$ on $(\mathrm{F} 2)$ & 0.0555 \\
\hline GooF & 1.128 \\
\hline$\Delta \rho \min \left(-\mathrm{e} . \AA^{-3}\right)$ & -0.54 \\
\hline$\Delta \rho \max \left(\mathrm{e} . \AA^{-3}\right)$ & 0.82 \\
\hline
\end{tabular}

The antiperovskite module $\left\{\left[\mathrm{F}_{2} \mathrm{OCa}_{12}\right]\left(\mathrm{SiO}_{4}\right)_{4}\right\}^{4+}$ consists of columns formed by Ca-triplets $\mathrm{Ca}_{3} \mathrm{O}_{14}$, rotated relative to each other by $60^{\circ}$ (Figure $4 \mathrm{~A}, \mathrm{~B}$ ). Ca-triplets form four layers with $\left(\mathrm{SiO}_{4}\right)^{4-}$ tetrahedra in structural cavities (Figure $4 \mathrm{C}$ ). The structure of hatrurite $\left(\mathrm{Ca}_{3} \mathrm{SiO}_{5}\right)$ shows similar antiperovskite modules $[1,18,19]$.

Raman and electron microprobe data (Figure 2, Table 1, L15) indicate the presence of small amounts of $\left(\mathrm{CO}_{3}\right)^{2-}$ in ariegilatite, replacing $\left(\mathrm{PO}_{4}\right)^{3-}$ groups. Refinement of the occupancy factor of P1 (Table 3) shows a small reduction from full occupation (89\%), which is in agreement with the substitution by $\mathrm{CO}_{3}$. The structural formula of ariegilatite calculated assuming a balanced charge, $\mathrm{BaCa}_{12}\left(\mathrm{SiO}_{4}\right)_{4}\left\{\left[\left(\mathrm{PO}_{4}\right)_{0.89 \square 0.11}\right]\left(\mathrm{CO}_{3}\right)_{0.11}\right\}_{2} \mathrm{~F}_{1.78} \mathrm{O}_{1.22}$, is close to the empirical formula obtained from microprobe analyses (Table 1, L15). However, the exact location and orientation of the planar $\mathrm{CO}_{3}$ group cannot be determined from diffraction data. Most likely, replacement of $\left(\mathrm{PO}_{4}\right)^{3-}$ by planar $\left(\mathrm{CO}_{3}\right)^{2-}$ groups takes place the same way as in stracherite: $\mathrm{CO}_{3}$ triangles are randomly located along one of the faces of the replaced tetrahedra excluding the face parallel to (001) [10]. Replacement of $\left(\mathrm{PO}_{4}\right)^{3-}$ by $\left(\mathrm{CO}_{3}\right)^{2-}$ in ariegilatite takes place according to the schemes: $\mathrm{Ca}^{2+}+\left(\mathrm{PO}_{4}\right)^{3-} \leftrightarrow \mathrm{Na}^{+}+\left(\mathrm{CO}_{3}\right)^{2-}$, $2\left(\mathrm{PO}_{4}\right)^{3-} \leftrightarrow\left(\mathrm{SiO}_{4}\right)^{4-}+\left(\mathrm{CO}_{3}\right)^{2-}$ and $\left(\mathrm{PO}_{4}\right)^{3-}+\mathrm{F}^{-} \leftrightarrow\left(\mathrm{CO}_{3}\right)^{2-}+\mathrm{O}^{2-}$.

As ariegilatite occurs only in tiny amounts, and the crystals contain numerous inclusions of other phases, useful X-ray powder diffraction data could not be collected. However, a powder pattern was calculated (Table S1) using the model derived from the single-crystal structure refinements. 
Table 3. Atom coordinates, $U_{\text {eq }}\left(\AA^{2}\right)$ values for ariegilatite.

\begin{tabular}{cccccc}
\hline Atom & $\mathbf{x} / \boldsymbol{a}$ & $\mathbf{y} / \boldsymbol{b}$ & $\mathbf{z} / \boldsymbol{c}$ & sof & Ueq \\
\hline Ba1 & 0 & 0 & 0 & 1 & $0.01731(9)$ \\
Ca1 & $0.16368(3)$ & $0.83632(3)$ & $0.39407(2)$ & 1 & $0.01059(12)$ \\
Ca2 & $0.15534(3)$ & $0.84466(3)$ & $0.53179(2)$ & 1 & $0.01036(12)$ \\
P1 & 0 & 0 & $0.67473(2)$ & $0.892(4)$ & $0.0085(2)$ \\
Si1 & 0 & 0 & $0.20615(2)$ & 1 & $0.00650(17)$ \\
Si2 & 0 & 0 & $0.08404(2)$ & 1 & $0.00723(17)$ \\
O1 & $0.55031(11)$ & $0.44969(11)$ & $0.64495(3)$ & 1 & $0.0148(4)$ \\
O2 & $0.12532(10)$ & $0.87468(10)$ & $0.19359(3)$ & 1 & $0.0130(4)$ \\
O3 & $0.12681(11)$ & $0.87319(11)$ & $0.07376(3)$ & 1 & $0.0140(4)$ \\
O4 & 0 & 0 & $0.36244(5)$ & 1 & $0.0125(5)$ \\
O5 & 0 & 0 & $0.75407(5)$ & 1 & $0.0132(5)$ \\
O6 & 0 & 0 & $0.12420(5)$ & 1 & $0.0113(5)$ \\
O7 & 0 & 0 & 0.5 & 1 & $0.0057(6)$ \\
F1 & 0 & 0 & $0.43179(4)$ & 1 & $0.0134(4)$ \\
\hline
\end{tabular}

Table 4. Anisotropic displacement parameters $U^{\mathrm{ij}}$ for ariegilatite.

\begin{tabular}{ccccccc}
\hline Atom & $\boldsymbol{U}^{\mathbf{1 1}}$ & $\boldsymbol{U}^{\mathbf{2 2}}$ & $\boldsymbol{U}^{\mathbf{3 3}}$ & $\boldsymbol{U}^{\mathbf{2 3}}$ & $\boldsymbol{U}^{\mathbf{1 3}}$ & $\boldsymbol{U}^{\mathbf{1 2}}$ \\
\hline Ba1 & $0.01604(12)$ & $0.01604(12)$ & $0.01985(15)$ & $0.00802(6)$ & 0 & 0 \\
Ca1 & $0.00850(14)$ & $0.00850(14)$ & $0.01499(17)$ & $0.00441(12)$ & $0.00013(5)$ & $-0.00013(5)$ \\
Ca2 & $0.00855(14)$ & $0.00855(14)$ & $0.01337(17)$ & $0.00382(12)$ & $-0.00066(5)$ & $0.00066(5)$ \\
P1 & $0.0087(3)$ & $0.0087(3)$ & $0.0082(4)$ & $0.00437(15)$ & 0 & 0 \\
Si1 & $0.0053(2)$ & $0.0053(2)$ & $0.0089(3)$ & $0.00264(11)$ & 0 & 0 \\
Si2 & $0.0063(2)$ & $0.0063(2)$ & $0.0091(3)$ & $0.00316(11)$ & 0 & 0 \\
O1 & $0.0156(4)$ & $0.0156(4)$ & $0.0144(5)$ & $0.0088(5)$ & $0.0010(2)$ & $-0.0010(2)$ \\
O2 & $0.0113(4)$ & $0.0113(4)$ & $0.0194(6)$ & $0.0079(5)$ & $0.0009(2)$ & $-0.0009(2)$ \\
O3 & $0.0107(4)$ & $0.0107(4)$ & $0.0220(6)$ & $0.0065(5)$ & $0.0015(2)$ & $-0.0015(2)$ \\
O4 & $0.0136(6)$ & $0.0136(6)$ & $0.0103(8)$ & $0.0068(3)$ & 0 & 0 \\
O5 & $0.0147(6)$ & $0.0147(6)$ & $0.0102(8)$ & $0.0074(3)$ & 0 & 0 \\
O6 & $0.0114(6)$ & $0.0114(6)$ & $0.0110(8)$ & $0.0057(3)$ & 0 & 0 \\
O7 & $0.0059(7)$ & $0.0059(7)$ & $0.0054(10)$ & $0.0030(4)$ & 0 & 0 \\
F1 & $0.0131(5)$ & $0.0131(5)$ & $0.0140(8)$ & $0.0066(3)$ & 0 & 0 \\
\hline
\end{tabular}

Table 5. Selected interatomic distances ( $)$ for ariegilatite.

\begin{tabular}{cccccccc}
\hline Atom & -Atom & Distance & & Atom & -Atom & Distance & \\
\hline Ba1 & -O1 & $2.8346(10)$ & $\times 6$ & P1 & -O4 & $1.535(2)$ & \\
& mean & 2.8346 & & & $-O 1$ & $1.5484(11)$ & $\times 3$ \\
\hline Ca1 & -O5 & $2.3735(11)$ & & & mean & 1.5451 & \\
& -O3 & $2.4006(9)$ & $\times 2$ & & bvs & $4.859(9)$ & \\
& -O4 & $2.4128(12)$ & & Si1 & -O2 & $1.6374(10)$ & $\times 3$ \\
& -O1 & $2.4628(12)$ & $\times 2$ & & -O5 & $1.643(2)$ & \\
& F1 & $2.5577(12)$ & & & mean & 1.6388 & \\
& mean & 2.4387 & & & bvs & $3.843(7)$ & \\
& bvs & $1.921(2)$ & & Si2 & -O3 & $1.6279(10)$ & $\times 3$ \\
\hline Ca2 & -O6 & $2.2495(5)$ & & & -O6 & $1.659(2)$ & \\
& -F1 & $2.4431(12)$ & & & mean & 1.6356 & \\
& -O2 & $2.3301(8)$ & $\times 2$ & & bvs & $3.879(7)$ & \\
& -O7 & $2.3303(5)$ & & O7 & -Ca2 & $2.3303(5)$ & $\times 6$ \\
& -O2 & $2.5114(15)$ & & & mean & 2.3303 & \\
& -O3 & $2.6028(15)$ & & & bvs & $2.248(1)$ & \\
& mean & 2.3996 & & F1 & -Ca1 & $2.5577(12)$ & $\times 3$ \\
& bvs & $2.196(2)$ & & & -Ca2 & $2.4431(12)$ & $\times 3$ \\
& bvs & $1.024(1)$ & & & mean & 2.5004 & \\
\hline
\end{tabular}



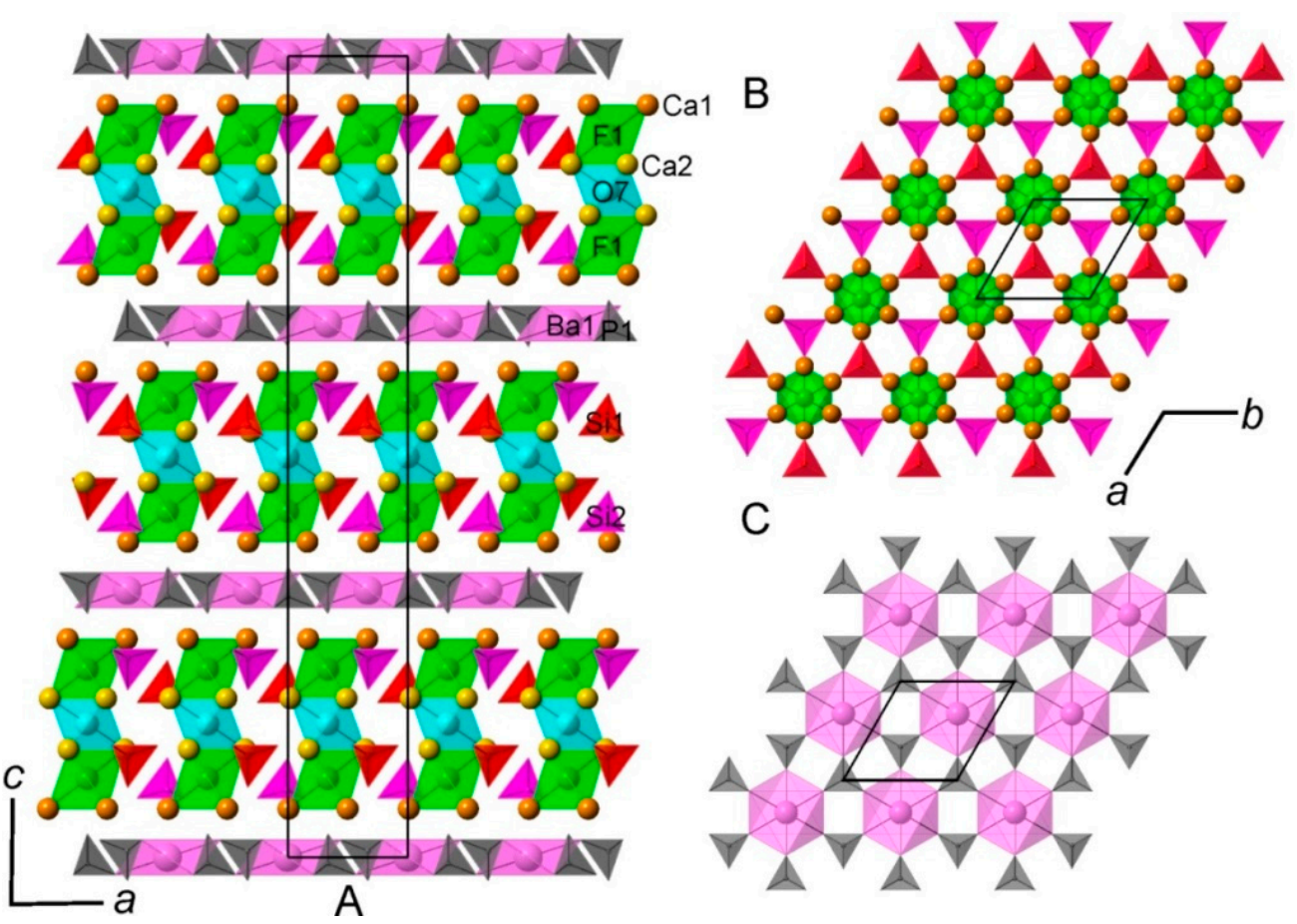

Figure 3. (A) The structure of ariegilatite, $\mathrm{BaCa}_{12}\left(\mathrm{SiO}_{4}\right)_{4}\left(\mathrm{PO}_{4}\right)_{2} \mathrm{~F}_{2} \mathrm{O}$, described as a $1: 1$ stacking of the two modules $\left\{\left[\mathrm{F}_{2} \mathrm{OCa}_{12}\right]\left(\mathrm{SiO}_{4}\right)_{4}\right\}^{4+}$ and $\left\{\mathrm{Ba}\left(\mathrm{PO}_{4}\right)_{2}\right\}^{4-}$ along (001). Atoms F1 and $\mathrm{O} 7$ are surrounded by six $\mathrm{Ca}$ atoms and form face-sharing octahedra (green and blue). (B) Module $\left\{\left[\mathrm{F}_{2} \mathrm{OCa}_{12}\right]\left(\mathrm{SiO}_{4}\right)_{4}\right\}^{4+}$ consisting of close-packed seven-fold coordinated $\mathrm{Ca}$ (dark yellow filled circles) with $\left(\mathrm{SiO}_{4}\right)$ filling the gaps (red and purple tetrahedra). (C) The $\left\{\mathrm{Ba}\left(\mathrm{PO}_{4}\right)_{2}\right\}^{4-}$ module is characterized by $\left(\mathrm{PO}_{4}\right)$ tetrahedra (lilac) connected to six-fold coordinated Ba (purple translucent octahedra).

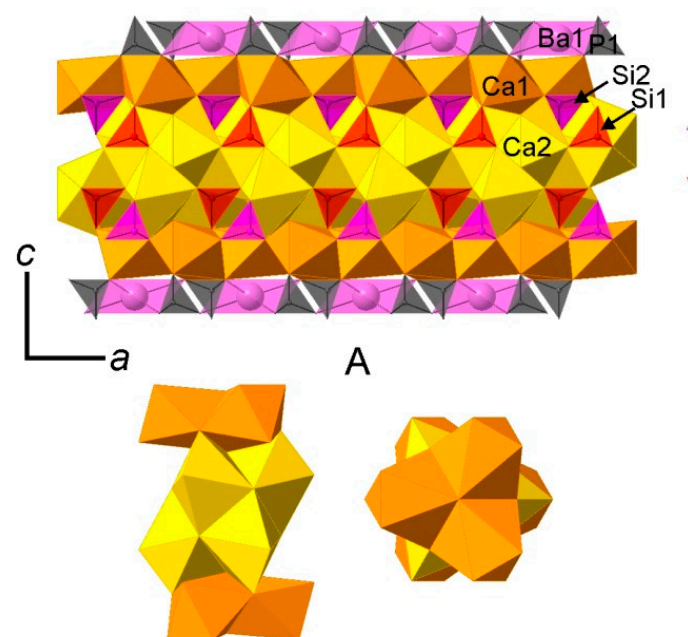

B

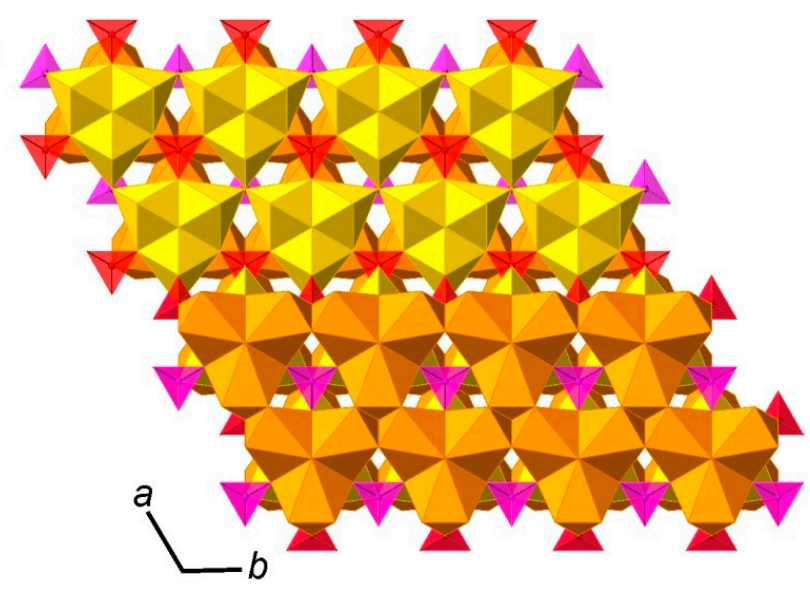

C

Figure 4. Using a cation-centered representation of the ariegilatite structure $(\mathbf{A}-\mathrm{C})$, the antiperovskite module can be decomposed into four layers assembled of triplets of Ca coordination polyhedra. Structural voids within each layer accommodate the silicate tetrahedra. Perpendicular through the layers, columns of four Ca-triplets can be identified. These are shown in a projection along c and [010] in (B). The layers of Ca-triplets are connected by common anions in octahedral coordination: $\mathrm{Ca} 1$ and $\mathrm{Ca} 2$ layers are sharing F1; Ca2 and Ca2 are connected by O7. In (C), two layers of Ca-triplets are removed at the top half of the image. 


\subsection{Occurrences of Ariegilatite in Other Localities of the Hatrurim Complex Rocks}

Ariegilatite occurs both in larnite and spurrite rocks of the Hatrurim Complex, whereas minerals of the nabimusaite-dargaite series are found in larnite rocks only [1,2]. The first samples of ariegilatite were found in 2011 by M. Murashko in larnite pebble, in the northern part of the Siwaqa pyrometamorphic rock area, $80 \mathrm{~km}$ south of Amman, Jordan. Daba-Siwaga is the largest area of the Hatrurim Complex within the Dead Sea rift region [20-22]. In this samples, ariegilatite is associated with larnite, gehlenite, spinel, fluormayenite, fluorapatite, perovskite and a bredigite-like Ba-bearing mineral (Figure 5A). It forms poikilitic crystals up to $0.25 \mathrm{~mm}$ in size and fine reaction rims on fluorapatite (Figure $5 \mathrm{~A}$ ). The mean ariegilatite composition from larnite rock is $\left(\mathrm{Ba}_{0.96} \mathrm{~K}_{0.02} \mathrm{Na}_{0.02}\right)_{\Sigma 1}\left(\mathrm{Ca}_{11.75} \mathrm{Mg}_{0.12} \mathrm{Fe}^{2+}{ }_{0.08} \mathrm{Mn}^{2+}{ }_{0.05}\right)_{\Sigma 12}\left(\mathrm{Si}_{3.86} \mathrm{Ti}^{4+}{ }_{0.05} \mathrm{Fe}^{3+}{ }_{0.05} \mathrm{Al}_{0.04}\right)_{\Sigma 4}\left(\mathrm{P}_{1.80} \mathrm{~S}^{6+}{ }_{0.09} \mathrm{Si}_{0.07}\right.$ $\left.\mathrm{V}^{5+}{ }_{0.04}\right)_{\Sigma 2} \mathrm{~F}_{2.09} \mathrm{O}_{0.91}$ (Table 1, YV595); here, $\mathrm{Mg}$ is slightly increased in comparison to the holotype specimen (Table 1, L15). The Raman spectrum of ariegilatite from larnite rocks in Jordan shows no characteristic bands of $\left(\mathrm{CO}_{3}\right)^{2-}$ groups (Figure 2, YV595). Furthermore, the calculation of the stoichiometric formula does not indicate the presence of $\left(\mathrm{CO}_{3}\right)^{2-}$ (Table 1, YV595).

Investigation of spurrite rocks (+calcite, fluorapatite, fluormayenite-fluorkyuygenite, brownmillerite and periclase) occurring within a 1-2-km radius from the place, where the larnite pebble was found at the location in Siwaqa, revealed ariegilatite metacrysts 30-50 $\mu \mathrm{m}$ in size (Figure 3B). These rocks are locally enriched in sphalerite and oldhamite and are interspersed with calcite veins of $1-2 \mathrm{~mm}$ in thickness. As well as the holotype material, ariegilatite from spurrite rocks of Jordan contains about $0.15-0.17\left(\mathrm{CO}_{3}\right)^{2-} p f u$ (Table 1 , SS20b and SS27A). The presence of $\left(\mathrm{CO}_{3}\right)^{2-}$ is also confirmed by Raman spectroscopy (Figure 2, SS20b and SS27A).
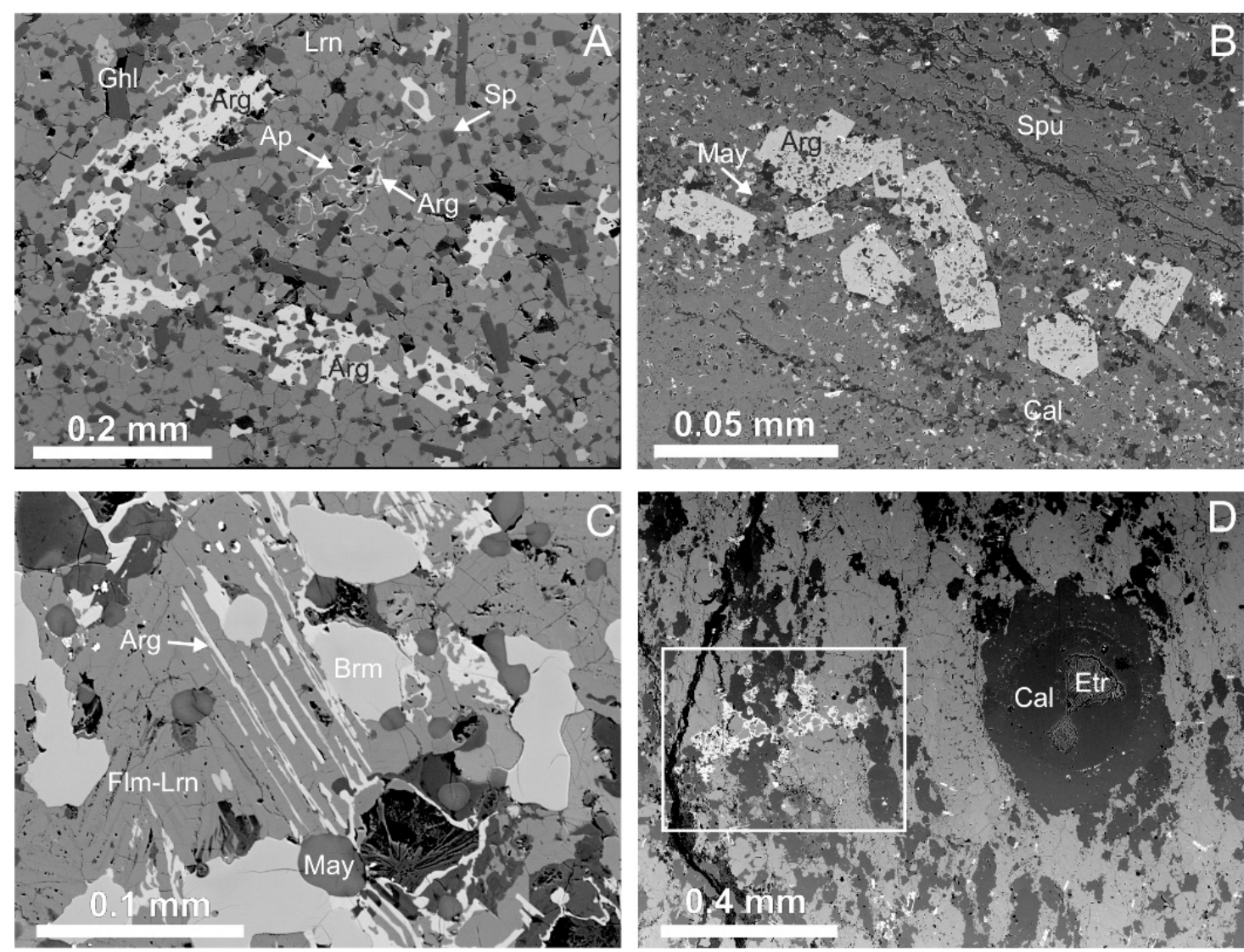

Figure 5. Cont. 

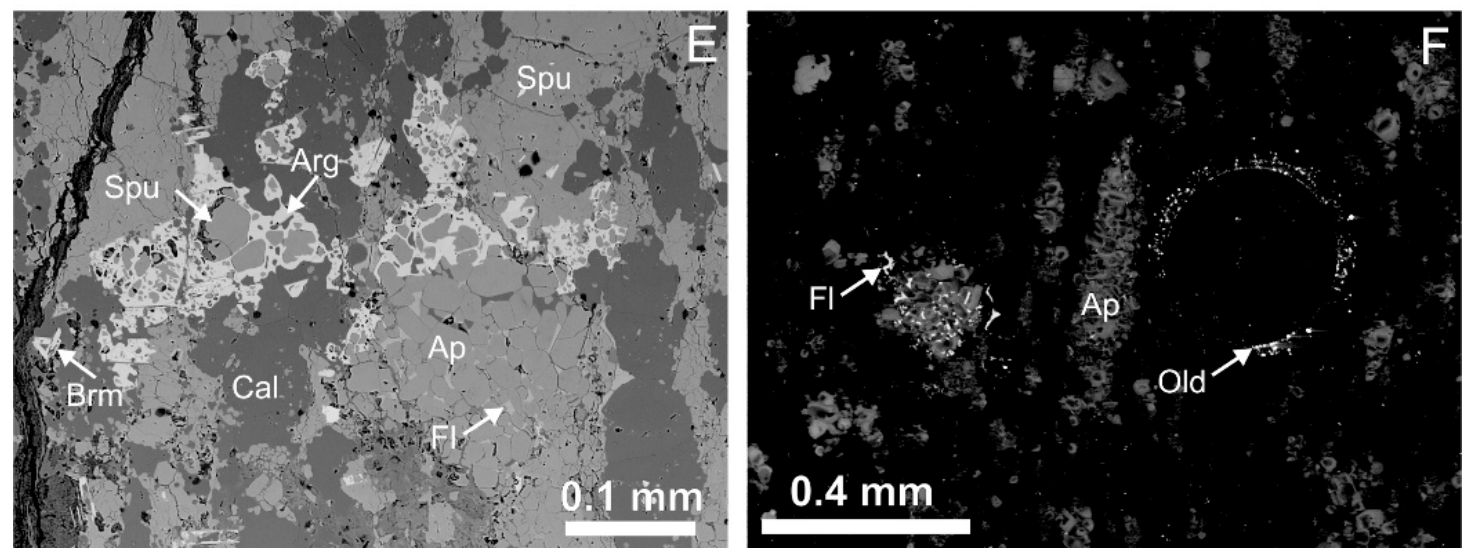

Figure 5. Poikilitic crystals of ariegilatite in gehlenite-larnite rock (A), where it also forms reaction rims on fluorapatite, Daba-Siwaqa, Jordan (Sample No. YV595); poikilitic crystals of ariegilatite in spurrite rock (B), Daba-Siwaqa, Jordan (SS20b); replacement of flamite by ariegilatite with exsolution structures (C), Ma'ale Adummim, Palestinian Autonomy (MA5b); apatite-spurrite rock with ariegilatite and channels filled with calcite (D). The fragment magnified in (E) is shown in the frame; Gurim Anticline, Negev Desert, Israel (IS129); xenomorphic aggregates of ariegilatite with spurrite inclusions (E); cathodoluminescence image of the area shown in $(\mathbf{D}, \mathbf{F})$, round shape of the channel, the walls of which are incrusted by oldhamite, having intensive luminescence. Arg = ariegilatite, Ap = fluorapatite, $\mathrm{Brm}=$ brownmillerite, $\mathrm{Cal}=$ calcite, $\mathrm{Etr}=$ ettringite, $\mathrm{Flm}=$ flamite, $\mathrm{Fl}=$ fluorite, $\mathrm{Ghl}=$ gehlenite, Lrn = larnite; May = fluormayenite-fluorkyuygenite, Old = oldhamite, $\mathrm{Sp}=$ spinel, $\mathrm{Spu}=$ spurrite.

Ariegilatite was also found in the territory of Palestinian Autonomy in an outcrop near the Jerusalem-Dead Sea highway close to Ma'ale Adummim. At this location, the mineral was detected in altered flamite rocks (+fluormayenite-fluorkyuygenite, brownmillerite, fluorapatite, gehlenite) with heterogeneous development of jasmundite. Flamite is an analog of the synthetic phase $\alpha-\mathrm{Ca}_{2} \mathrm{SiO}_{4}$ stabilized by $\mathrm{K}, \mathrm{Na}$ and $\mathrm{P}$ impurities $[23,24]$. Flamite usually shows exsolution structures with individual lamellas enriched in P [24]. Ariegilatite replaces flamite lamellas with increased phosphorus content (Figure 5C) and exhibits a mean composition of $\left(\mathrm{Ba}_{0.97} \mathrm{~K}_{0.02} \mathrm{Na}_{0.01}\right)_{\Sigma 1}\left(\mathrm{Ca}_{11.87} \mathrm{Na}_{0.07}\right.$ $\left.\mathrm{Mg}_{0.03} \mathrm{Fe}^{2+}{ }_{0.03}\right)_{\Sigma 12}\left(\mathrm{Si}_{3.35} \mathrm{P}_{0.50} \mathrm{Al}_{0.13} \mathrm{Ti}^{4+}{ }_{0.02}\right)_{\Sigma 4}\left(\mathrm{P}_{1.72} \mathrm{~S}^{6+}{ }_{0.21} \mathrm{C}_{0.05} \mathrm{~V}^{5+}{ }_{0.02}\right)_{\Sigma 2} \mathrm{~F}_{1.48} \mathrm{O}_{1.52}$, and it is characterized by increased $\left(\mathrm{SO}_{4}\right)^{2-}$ content (Table 1, MA5b); accordingly, its Raman spectrum shows a strong $v_{1}\left(\mathrm{SO}_{4}\right)^{2-}$ band near $995 \mathrm{~cm}^{-1}$ (Figure 2, MA5b).

Ariegilatite samples showing the highest $\left(\mathrm{CO}_{3}\right)^{2-}$ contents were detected in fluorapatite-calcite-spurrite rocks in the Negev Desert near the junction of Hatrurim (Gurim Anticline, Hatrurim Basin), Israel (Figure 5D,E). In these rocks, also cuspidine, fluorite, brownmillerite, chlormayenite, periclase, sphalerite, millerite, U-bearing lakargiite, vorlanite and oldhamite are identified. The empirical formula of ariegilatite from this locality is $\left(\mathrm{Ba}_{0.97} \mathrm{~K}_{0.01} \mathrm{Na}_{0.02}\right)_{\Sigma 1}$ $\left(\mathrm{Ca}_{11.76} \mathrm{Na}_{0.24}\right)_{\Sigma 12}\left(\mathrm{Si}_{3.97} \mathrm{Al}_{0.03}\right)_{\Sigma 4}\left(\mathrm{P}_{1.53} \mathrm{C}_{0.46} \mathrm{Si}_{0.01}\right)_{\Sigma 2} \mathrm{~F}_{1.85} \mathrm{O}_{1.15}$ (Table 1, IS129). Its Raman spectrum reveals a characteristic band of $\left(\mathrm{CO}_{3}\right)^{2-}$ groups (Figure 2, IS129). The $\left(\mathrm{CO}_{3}\right)^{2-}$ groups can be incorporated according to two schemes: $\left(\mathrm{PO}_{4}\right)^{3-}+\mathrm{Ca}^{2+} \leftrightarrow\left(\mathrm{CO}_{3}\right)^{2-}+\mathrm{Na}^{+}$and $\left(\mathrm{PO}_{4}\right)^{3-}+\mathrm{F}^{-} \leftrightarrow$ $\left(\mathrm{CO}_{3}\right)^{2-}+\mathrm{O}^{2-}$. Interestingly, in spurrite rock, an ariegilatite relic net of dendrite channels (with an oval cross-section of $0.2-0.4 \mathrm{~mm}$ in diameter) filled with coarse-crystalline calcite was observed. Walls of the channels are incrusted by small oldhamite crystals up to $1-2 \mu \mathrm{m}$ in size. Thanks to intensive oldhamite cathodoluminescence, these channels are well visible within the background of the weakly-luminescent minerals of spurrite rocks (Figure 5F). 


\section{Discussion}

Ariegilatite exhibits an ordered anion distribution. Fluorine and oxygen were assigned to the anion sites utilizing the results of bond valence sum (BVS, Table 5) calculations. Oxygen resides in the center (Figure 3A, blue) and fluorine on the two outer octahedral sites (Figure 3A, green) of the antiperovskite module. New BVS calculations revealed the same preference in nabimusaite, where one fluorine atom seems to be distributed over the two outer octahedral sites, in contrast to what has been reported before $[1,2,25]$. Fluorine may enter the central octahedra when the charge is compensated as, for example, in arctite $\mathrm{BaCa}_{7} \mathrm{Na}_{5}\left(\mathrm{PO}_{4}\right)_{6} \mathrm{~F}_{3}[6,8]$, where $\mathrm{Ca}$ atoms (of the central octahedra) are substituted by sodium. Similar behavior is observed in the isostructural synthetic compound $\mathrm{Ca}_{5.45} \mathrm{Li}_{3.55}\left[\mathrm{SiO}_{4}\right]_{3} \mathrm{O}_{0.45} \mathrm{~F}_{1.55}$ [26], where the outer octahedral sites are occupied by fluorine, whereas the inner site seems to be shared by oxygen and fluorine. For charge compensation, the Ca-sites coordinating the inner octahedral site are partly substituted by lithium.

The main schemes of isomorphism, unifying minerals of the nabimusaite group and arctite are $\mathrm{Ca}^{2+}+\mathrm{SiO}_{4}{ }^{4-}=\mathrm{Na}^{+}+\mathrm{PO}_{4}{ }^{3-}$ and $\mathrm{Ca}^{2+}+\mathrm{O}^{2-}=\mathrm{Na}^{+}+\mathrm{F}^{-}$. Arctite was the first mineral with a hexagonal intercalated antiperovskite structure to be discovered. We propose to distinguish an arctite super group (hexagonal intercalated antiperovskites), which at present combines two silicate groups: nabimusaite (nabimusaite, dargaite, ariegilatite, with triple-layer antiperovskite modules) and zadovite (zadovite, aradite, gazeevite, stracherite, with single-layer antiperovskite modules) and arctite, as an ungrouped phosphate.

According to Krivovichev [19], polyphite, $\mathrm{Na}_{6}\left(\mathrm{Na}_{4} \mathrm{Ca}_{2}\right)_{2} \mathrm{Na}_{2} \mathrm{Ti}_{2} \mathrm{Na}_{2} \mathrm{Ti}_{2}\left(\mathrm{Si}_{2} \mathrm{O}_{7}\right)_{2}\left(\mathrm{PO}_{4}\right)_{6} \mathrm{O}_{4} \mathrm{~F}_{4}$ [27], also belongs to the hexagonal intercalated antiperovskites. However, we propose that polyphite, the structurally-related sobolevite, $\mathrm{Na}_{12} \mathrm{Ca}(\mathrm{NaCaMn}) \mathrm{Ti}_{2}(\mathrm{Ti}, \mathrm{Mn})\left[\mathrm{Si}_{2} \mathrm{O}_{7}\right]_{2}\left(\mathrm{PO}_{4}\right)_{4} \mathrm{O}_{3} \mathrm{~F}_{3}$, and quadruphite, $\mathrm{Na}_{14} \mathrm{Ca}_{2} \mathrm{Ti}_{4}\left[\mathrm{Si}_{2} \mathrm{O}_{7}\right]_{2}\left(\mathrm{PO}_{4}\right)_{4} \mathrm{O}_{4} \mathrm{~F}_{2}$ [27], should not be considered in the arctite super group. In these structures, columns of anion-centered octahedra form continuous layers parallel to titanium-silicate sheets. The proposed arctite super group exhibits $A\left(T \mathrm{O}_{4}\right)$ layers, which are perpendicular to the "antiperovskite columns" (Figure 3A).

So far, ariegilatite shows the highest fluorine content ( $>3 \mathrm{wt} \%$, Table 1$)$ of all members of the nabimusaite group. It occurs in spurrite, as well as in larnite rocks (Figures 1 and 5). Ariegilatite from spurrite rocks contain significant amounts of $\left(\mathrm{CO}_{3}\right)^{2-}$ (Table 1). It forms relatively large poikilitic crystals standing out against a fine-grained matrix and grows after fluorapatite (Figures 1 and 5). Minerals of the dargaite-nabimusaite series (and also gazeevite), as well as minerals of the ternesite-silicocarnotite series and jasmundite are high-temperature alteration products of minerals of an early clinker-like association of larnite rocks. These alterations took place under the influence of pyrometamorphism by-products, such as gases and fluids generated by closely-spaced combustion foci $[1,28,29]$.

In our understanding, ariegilatite, as well as associated stracherite are the products of high-temperature alteration of early pyrometamorphic rocks with increased phosphorus (fluorapatite) and decreased sulfur (absence of fluorellestadite and ye'elimite) contents under the influence of high-fluorine, $\mathrm{CO}_{2}$-bearing fluids/gases. Transport of these fluids/gases is realized through cracks and micro-channel nets (Figure 5F), which may have existed for a long time in heated pyrometamorphic rocks.

Supplementary Materials: The following are available online at http:/ /www.mdpi.com/2075-163X/8/3/19/s1, Table S1: Calculated powder pattern for ariegilatite. Intensities were calculated using the software JANA2006 [30].

Acknowledgments: The investigations were partially supported by the National Science Centre of Poland, Grant No. 2016/23/B/ST10/00869. We would like to thank Jakub Kaminski for important technical assistance during the diffraction experiments at X06DA. 
Author Contributions: Evgeny V. Galuskin, Biljana Krüger, Irina O. Galuskina and Hannes Krüger wrote the paper. Evgeny V. Galuskin, Irina O. Galuskina and Yevgeny Vapnik took part in the field works, by the results of which ariegilatite was discovered. Mikhail Murashko found the first specimen with ariegilatite in Jordan. Evgeny V. Galuskin and Irina O. Galuskina performed petrological investigations, measurement of ariegilatite composition from different localities, Raman and optical studies and also selected grains for structural investigations. Biljana Krüger and Hannes Krüger performed SC XRD investigation and refined ariegilatite structure. Justyna A. Wojdyla helped in single-crystal structural investigations using synchrotron radiation at the super-bending magnet beamline X06DA at the Swiss Light Source.

Conflicts of Interest: The authors declare no conflict of interest.

\section{References}

1. Galuskin, E.V.; Gfeller, F.; Armbruster, T.; Galuskina, I.O.; Vapnik, Ye.; Murashko, M.; Wodyka, R.; Dzierżanowski, P. New minerals with modular structure derived from hatrurite from the pyrometamorphic Hatrurim Complex, Part I: Nabimusaite, $\mathrm{KCa}_{12}\left(\mathrm{SiO}_{4}\right)_{4}\left(\mathrm{SO}_{4}\right)_{2} \mathrm{O}_{2} \mathrm{~F}$, from larnite rock of the Jabel Harmun, Palestinian Autonomy, Israel. Mineral. Mag. 2015, 79, 1061-1072. [CrossRef]

2. Galuskina, I.O.; Gfeller, F.; Galuskin, E.V.; Armbruster, T.; Vapnik, Ye.; Dulski, M.; Gardocki, M.; Jeżak, L.; Murashko, M. New minerals with modular structure derived from hatrurite 1 from the pyrometamorphic rocks, part IV: Dargaite, $\mathrm{CaCa}_{12}\left(\mathrm{SiO}_{4}\right)_{4}\left(\mathrm{SO}_{4}\right)_{2} \mathrm{O}_{3}$, from Nahal Darga, Palestinian Autonomy. Mineral. Mag. 2018, 82. in press.

3. Bentor, Y.K. (Ed.) Israel. In Lexique Stratigraphique International, Asie; CNRS: Paris, France, 1960; Volume III, Chapter 10.2.

4. Gross, S. The Mineralogy of the Hatrurim Formation, Israel; Geological Survey of Israel: Jerusalem, Israel, 1977.

5. Vapnik, Y.; Sharygin, V.V.; Sokol, E.V.; Shagam, R. Paralavas in a combustion metamorphic complex: Hatrurim Basin, Israel. Rev. Eng. Geol. 2007, 18, 1-21.

6. Sokolova, E.V.; Yamnova, N.A.; Egorov-Tismenko, Y.K.; Khomyakov, A.P. The crystal structure of a new sodium-calcium-barium phosphate of $\mathrm{Na}, \mathrm{Ca}$ and $\mathrm{Ba}\left(\mathrm{Na}_{5} \mathrm{Ca}\right) \mathrm{Ca}_{6} \mathrm{Ba}\left(\mathrm{PO}_{4}\right)_{6} \mathrm{~F}_{3}$. Dokl. Akad. Nauk SSSR 1984, 274, 78-83.

7. Galuskin, E.V.; Gfeller, F.; Galuskina, I.O.; Armbruster, T.; Krzatała, A.; Vapnik, Ye.; Kusz, J.; Dulski, M.; Gardocki, M.; Gurbanov, A.G.; et al. New minerals with a modular structure derived from hatrurite from the pyrometamorphic rocks. Part III. Gazeevite, $\mathrm{BaCa}_{6}\left(\mathrm{SiO}_{4}\right)_{2}\left(\mathrm{SO}_{4}\right)_{2} \mathrm{O}$, from Israel and the Palestine Autonomy, South Levant, and from South Ossetia, Greater Caucasus. Mineral. Mag. 2017, 81, 499-513. [CrossRef]

8. Sokolova, E.; Hawthorne, F.C. The crystal chemistry of the $\left[\mathrm{M}_{3} \varphi_{11-14}\right]$ trimeric structures: From hyperagpaitic complexes to saline lakes. Can. Mineral. 2001, 39, 1275-1294. [CrossRef]

9. Galuskin, E.V.; Gfeller, F.; Galuskina, I.O.; Pakhomova, A.; Armbruster, T.; Vapnik, Y.; Włodyka, R.; Dzierżanowski, P.; Murashko, M. New minerals with modular structure derived from hatrurite from the pyrometamorphic Hatrurim Complex, Part II: Zadovite, $\mathrm{BaCa}_{6}\left[\left(\mathrm{SiO}_{4}\right)\left(\mathrm{PO}_{4}\right)\right]\left(\mathrm{PO}_{4}\right)_{2} \mathrm{~F}$, and aradite, $\mathrm{BaCa}_{6}\left[\left(\mathrm{SiO}_{4}\right)\left(\mathrm{VO}_{4}\right)\right]\left(\mathrm{VO}_{4}\right)_{2} \mathrm{~F}$, from paralavas of the Hatrurim Basin, Negev Desert, Israel. Mineral. Mag. 2015, 79, 1073-1087. [CrossRef]

10. Galuskin, E.V.; Krüger, B.; Galuskina, I.O.; Krüger, H.; Vapnik, Ye.; Pauluhn, A.; Olieric, V. Stracherite, $\mathrm{BaCa}_{6}\left(\mathrm{SiO}_{4}\right)_{2}\left[\left(\mathrm{PO}_{4}\right)\left(\mathrm{CO}_{3}\right)\right] \mathrm{F}$, a first $\mathrm{CO}_{3}$-bearing intercalated hexagonal antiperovskite from Negev Desert, Israel. Amer. Mineral. 2018. under review.

11. Waltersperger, S.; Olieric, V.; Pradervand, C.; Glettig, W.; Salathe, M.; Fuchs, M.R.; Curtin, A.; Wang, X.; Ebner, S.; Panepucci, E.; et al. PRIGo: A new multi-axis goniometer for macromolecular crystallography. J. Synchrotron Radiat. 2015, 22, 895-900. [CrossRef] [PubMed]

12. Wojdyla, J.A.; Kaminski, J.W.; Panepucci, E.; Ebner, S.; Wang, X.; Gabadinho, J.; Wang, M. DA+ data acquisition and analysis software at the Swiss Light Source macromolecular crystallography beamlines. J. Synchrotron Radiat. 2018, 25, 293-303. [CrossRef] [PubMed]

13. Kabsch, W. XDS. Acta Crystallogr. D 2010, 166, 125-132. [CrossRef] [PubMed]

14. Sheldrick, G.M. A short history of SHELX. Acta Crystallogr. A 2008, 64, 112-122. [CrossRef] [PubMed]

15. Krüger, B.; Galuskin, E.V.; Galuskina, I.O.; Krüger, H.; Vapnik, Y.; Olieric, V.; Pauluhn, A. A potentially new mineral with a modular structure based on antiperovskite layers. Mitt. Österr. Miner. Ges. 2017, 163, 59.

16. Comodi, P.; Liu, Y. $\mathrm{CO}_{3}$ substitution in apatite: Further insight from new crystal-chemical data of Kasekere (Uganda) apatite. Eur. J. Mineral. 2000, 12, 965-974. [CrossRef] 
17. Banno, Y.; Miyawaki, R.; Momma, K.; Bunno, $\mathrm{M}$. $\mathrm{CO}_{3}$-bearing member of the hydroxylapatite-hydroxylellestadite series from Tadano, Fukushima Prefecture, Japan: $\mathrm{CO}_{3}-\mathrm{SO}_{4}$ substitution in the apatite-ellestadite series. Mineral. Mag. 2016, 80, 363-370. [CrossRef]

18. Jeffery, J.W. The crystal structure of tricalcium silicate. Acta Crystallogr. 1952, 5, 26-35. [CrossRef]

19. Krivovichev, S.V. Minerals with antiperovskite structure: A review. Z. Kristallogr. 2008, 223, 109-113. [CrossRef]

20. Geller, Y.I.; Burg, A.; Halicz, L.; Kolodny, Y. System closure during the combustion metamorphic "Mottled Zone" event, Israel. Chem. Geol. 2012, 334, 25-36. [CrossRef]

21. Novikov, I.; Vapnik, E.; Safonova, I. Mud volcano origin of the Mottled Zone, South Levant. Geosci. Front. 2013, 4, 597-619. [CrossRef]

22. Khoury, H.N.; Salameh, E.M.; Clark, I.D. Mineralogy and origin of surficial uranium deposits hosted in travertine and calcrete from central Jordan. Appl. Geochem. 2014, 43, 49-65. [CrossRef]

23. Sokol, E.V.; Seryotkin, Y.V.; Kokh, S.N.; Vapnik, Y.; Nigmatulina, E.N.; Goryainov, S.V.; Belogub, E.V.; Sharygin, V.V. Flamite, $(\mathrm{Ca}, \mathrm{Na}, \mathrm{K})_{2}(\mathrm{Si}, \mathrm{P}) \mathrm{O}_{4}$, a new mineral from ultra high temperature combustion metamorphic rocks, Hatrurim Basin, Negev Desert, Israel. Mineral. Mag. 2015, 79, 583-596. [CrossRef]

24. Gfeller, F.; Widmer, R.; Krüger, B.; Galuskin, E.V.; Galuskina, I.O.; Armbruster, T. The crystal structure of flamite and its relation to $\mathrm{Ca}_{2} \mathrm{SiO}_{4}$ polymorphs and nagelschmidtite. Eur. J. Mineral. 2015, 27, 755-769. [CrossRef]

25. Fayos, J.; Glasser, F.P.; Howie, R.A.; Lachowski, E.; Perez-Mendez, M. Structure of dodecacalcium potassium fluoride dioxide terasilicate bis (sulphate), $\mathrm{KF} .2\left[\mathrm{Ca}_{6}\left(\mathrm{SO}_{4}\right)\left(\mathrm{SiO}_{4}\right)_{2} \mathrm{O}\right]$ : A fluorine containing phase encountered in cement clinker production process. Acta Crystallogr. C 1985, C41, 814-816. [CrossRef]

26. Krüger, H. $\mathrm{Ca}_{5.45} \mathrm{Li}_{3.55}[\mathrm{SiO} 4]_{3} \mathrm{O}_{0.45} \mathrm{~F}_{1.55}$ and $\mathrm{Ca}_{7} \mathrm{~K}\left[\mathrm{SiO}_{4}\right]_{3} \mathrm{~F}_{3}$ : Single-crystal synthesis and structures of two trigonal oxyfluorides. Z. Kristallogr. 2010, 225, 418-424. [CrossRef]

27. Sokolova, E.; Hawthorne, F.C.; Khomyakov, A.P. Polyphite and sobolevite: Revision of their crystal structures. Can. Mineral. 2005, 43, 1527-1544. [CrossRef]

28. Galuskin, E.V.; Galuskina, I.O.; Gfeller, F.; Krüger, B.; Kusz, J.; Vapnik, Ye.; Dulski, M.; Dzierżanowski, P. Silicocarnotite, $\mathrm{Ca}_{5}\left[\left(\mathrm{SiO}_{4}\right)\left(\mathrm{PO}_{4}\right)\right]\left(\mathrm{PO}_{4}\right)$, a new "old" mineral from the Negev Desert, Israel, and the ternesite-silicocarnotite solid solution: Indicators of high-temperature alteration of pyrometamorphic rocks of the Hatrurim Complex, Southern Levant. Eur. J. Mineral. 2016, 28, 105-123. [CrossRef]

29. Galuskina, I.O.; Galuskin, E.V.; Prusik, K.; Vapnik, Ye.; Juroszek, R.; Jeżak, L.; Murashko, M. Dzierżanowskite, $\mathrm{CaCu}_{2} \mathrm{~S}_{2}-\mathrm{A}$ new natural thiocuprate from Jabel Harmun, Judean Desert, Palestine Autonomy, Israel. Mineral. Mag. 2017, 81, 1073-1085. [CrossRef]

30. Petříček, V.; Dušek, M.; Palatinus, L. Crystallographic computing system JANA2006: General features. Z. Kristallogr. 2014, 229, 345-352. [CrossRef] 